\title{
Video Article \\ Long-term Behavioral and Reproductive Consequences of Embryonic Exposure to Low-dose Toxicants
}

\author{
Mahlet D. Mersha ${ }^{1}$, Karla R. Sanchez ${ }^{2}$, Murali K. Temburni ${ }^{2}$, Harbinder S. Dhillon ${ }^{2}$ \\ ${ }^{1}$ Department of Neurology, Johns Hopkins School of Medicine \\ ${ }^{2}$ Department of Biological Sciences, Delaware State University
}

Correspondence to: Harbinder S. Dhillon at hsdhillon@desu.edu

URL: https://www.jove.com/video/56771

DOI: doi:10.3791/56771

Keywords: Developmental Biology, Issue 133, Bisphenol A, Bisphenol S, Caenorhabditis elegans, Endocrine Disrupting Compound, Development, Embryogenesis, Behavior, Habituation, Non-associative learning, Fecundity

Date Published: 3/6/2018

Citation: Mersha, M.D., Sanchez, K.R., Temburni, M.K., Dhillon, H.S. Long-term Behavioral and Reproductive Consequences of Embryonic Exposure to Low-dose Toxicants. J. Vis. Exp. (133), e56771, doi:10.3791/56771 (2018).

\section{Abstract}

Bisphenols, such as bisphenol A (BPA) and bisphenol S (BPS) are polymerizing agents widely used in the production of plastics and numerous everyday-use products. Based on their chemical structure and estradiol-like biological properties, they have been classified as endocrine disrupting compounds (EDC). Long-term exposure to EDCs, even at low doses, has been linked to various health defects including cancer, behavioral disorders and infertility, with greater vulnerability indicated during early developmental periods. Cellular and molecular studies with the genetically tractable nematode model Caenorhabditis elegans have demonstrated that exposure to BPA causes apoptosis, embryonic lethality and disruption in the DNA repair mechanisms. We have previously reported that exposure of $C$. elegans embryos to low doses of different bisphenols decreases fecundity. In addition, we have shown that the effects of exposure during the very early stages of development persist into adulthood as assayed by quantifying habituation behavior, a form of non-associative learning. Here, we provide detailed protocols for embryonic exposure to low-dose EDCs as well as the associated fecundity and anterior touch habituation assays, along with representative results.

\section{Video Link}

The video component of this article can be found at https://www.jove.com/video/56771/

Introduction

Exposure to environmental toxicants, particularly compounds those that tend to interfere with development, has been under careful scientific scrutiny in recent years. More than a thousand chemicals in everyday use are classified as endocrine disrupting compounds (EDC) ${ }^{1}$. Periods of rapid growth and development, which include embryonic, infant and childhood stages, have been noted to be particularly vulnerable to the deleterious effects to even low-dose EDC. Their effect have been shown to cause reproductive and neurodevelopmental disorders ${ }^{2}$. Per the US Environmental Protection Agency and US National Toxicology Program panel guidelines, a low dose may be defined as any dose below the level of one that has been reported to cause an observable biological change or damage ${ }^{3}$. Besides low-dose effects of individual EDCs, mixtures of various EDCs found at low concentrations in the environment have the potential to cause substantive cumulative effects ${ }^{4}$.

Bisphenol A (BPA or 4,4'-(propane-2,2-diyl)) is a polymerizing agent found in commonly used items such as water bottles, store receipts, dental sealants, and the linings of beverage and food cans ${ }^{5}$. Due to its structural similarity to 17- $\beta$ Estradiol $\left(E_{2}\right)$ and its affinity to the $E R \alpha$ and $E R \beta$ estrogen receptors, BPA has been classified as an endocrine disrupting chemical (EDC) $)^{5,6}$. Although weaker, BPA's affinity to estrogen receptors has been shown to affect the reproduction system of both sexes and disrupt neural functions at doses that are considered safe ${ }^{7,8}$. Changes in DNA methylation via epigenetically regulated mechanisms have been observed to cause long-term neuronal defects in mice exposed to $\mathrm{BPA}^{9}$. Specifically, BPA has also been implicated as a possible culprit for increased rates of hyperactivity, attention deficiency and increased sensitivity to drugs due to an increase seen in D1 dopamine receptors in the mouse limbic forebrain after chronic exposure , $^{9}$. Significant evidence on the deleterious effects of EDCs on human health is based on correlation studies focusing mainly on chronic exposure of populations to environmental toxicants even at low doses ${ }^{5}$; however, limitations in inferences from human studies and in manipulating experimental controls have been accepted while addressing criticisms of unsubstantiated hype ${ }^{11,12}$

Due to the conservation of Caenorhabditis elegans' genes with respect to mammals', including its steroid hormone-receptor genes, researchers have utilized this genetically tractable lab model to unravel the functional and mechanistic effects of EDCs ${ }^{13}$. Experiments with $C$. elegans have shown that these compounds such as BPA and BPS can cause apoptosis, embryonic lethality, disruption in the double-stranded DNA break repair mechanisms and neural function ${ }^{14,15,16}$.

Our lab has previously shown that even low-dose exposure limited to early embryogenesis leads to lowered fecundity with behavioral deficits in the surviving adults ${ }^{15}$. Habituation to a repeated stimulus is a form of non-associative learning in model systems, including $C$. elegans, and our methodology uses this form of non-associative learning as a behavioral output to assay the long-term effects of embryonic exposure to the toxicants ${ }^{17}$ Specifically, we provide detailed protocols for studying BPA exposure on C. elegans, including its immediate effects on embryonic 
lethality and long-term effects on adult behavior, with an overall schematic depicted in Figure 1. Representative results from fecundity and nonassociative learning assays are provided to highlight the effectiveness of our methodology.

\section{Protocol}

Protocols given below have been standardized to test the effects of BPA exposure during early embryogenesis, and may be modified for use with BPS, BPF or other EDCs and toxicants on C. elegans embryos.

\section{Material Setup}

1. Prepare $500 \mathrm{~mL}$ of Nematode Growth Media (NGM) ${ }^{18}$ media by dissolving $1.5 \mathrm{~g}$ of NaCl, $1.25 \mathrm{~g}$ of peptone, and $8.5 \mathrm{~g}$ of agar in water to 500 $\mathrm{mL}$. After autoclaving $\left(121^{\circ} \mathrm{C}, 15 \mathrm{PSI}, 20 \mathrm{~min}\right)$, add $0.5 \mathrm{~mL}$ of cholesterol $\left(5 \mathrm{mg} / \mathrm{mL}\right.$ in ethanol), $0.5 \mathrm{~mL}$ of $1 \mathrm{M} \mathrm{MgSO}_{4}, 0.5 \mathrm{~mL}$ of $1 \mathrm{M} \mathrm{CaCl}$. and $12.5 \mathrm{~mL}$ of $1 \mathrm{M}$ potassium phosphate buffer, $\mathrm{pH} 7.4$ (108.3 $\mathrm{g}$ of $\mathrm{KH}_{2} \mathrm{PO}_{4}, 35.6 \mathrm{~g}$ of $\mathrm{K}_{2} \mathrm{HPO}_{4}$, water to $1 \mathrm{~L}$ ).

2. Prepare hypochlorite solution by mixing $25 \mathrm{~mL}$ of $1 \mathrm{~N} \mathrm{KOH}, 4 \mathrm{~mL}$ of bleach, and $71 \mathrm{~mL}$ of water. Make this solution fresh

3. Prepare M9 Buffer by mixing $3 \mathrm{~g}$ of $\mathrm{KH}_{2} \mathrm{PO}_{4}, 6 \mathrm{~g}$ of $\mathrm{Na}_{2} \mathrm{HPO}_{4}, 5 \mathrm{~g}$ of NaCl, $5 \mathrm{~mL}$ of $1 \mathrm{M}$ of $\mathrm{MgSO}_{4}$, and water to $1 \mathrm{~L}$.

4. Prepare $\mathrm{S}$ Buffer ${ }^{18}$ by mixing $129 \mathrm{~mL}$ of $0.02 \mathrm{M} \mathrm{K}_{2} \mathrm{HPO}_{4}, 871 \mathrm{~mL}$ of $\mathrm{KH}_{2} \mathrm{PO}_{4}$, and $5.85 \mathrm{~g}$ of NaCl.

5. Sterilize $\mathrm{M} 9$ and $\mathrm{S}$ buffers by autoclaving.

6. Grow an overnight culture of Escherichia coli (OP 50 strain) in $20 \mathrm{~mL}$ of Luria Broth (LB).

7. Dissolve BPA in $10 \%$ ethanol to make a $1 \mathrm{mM}$ stock solution. Make subsequent dilutions of $0.1 \mu \mathrm{M}, 0.5 \mu \mathrm{M}, 1 \mu \mathrm{M}, 5 \mu \mathrm{M}$ and $10 \mu \mathrm{M}$ in $\mathrm{S}$ buffer.

NOTE: Dilution in the aqueous $S$ buffer greatly reduces the concentration of ethanol; for example, at the highest BPA concentration described in this protocol $(10 \mu \mathrm{M})$, the concentration of ethanol is $0.1 \% \mathrm{v} / \mathrm{v}$.

\section{C. elegans Culturing and Synchronization}

1. Pour the NGM media into $100 \mathrm{~mm}$ plates (about $25 \mathrm{~mL} /$ plate) and allow it to solidify. Once solidified, seed the plates by spreading $150 \mu \mathrm{L}$ of E. coli OP50 from the liquid culture grown overnight. Incubate plates at $37^{\circ} \mathrm{C}$ overnight.

2. The next day, transfer $\mathrm{N} 2$ worms onto the new plates by chunking an approximate slab of $1 \mathrm{~cm}$ square. Allow worms to grow for 3 days at 20 ${ }^{\circ} \mathrm{C}$ until the plate is populated with well-fed mature adults containing visible embryos.

3. To synchronize, wash each plate by pipetting up to $5 \mathrm{~mL}$ of $\mathrm{M} 9$ buffer across the plate. Transfer the liquid to a sterile $15 \mathrm{~mL}$ conical centrifuge tube.

NOTE: Synchronization is done to kill adult worms and collect embryos that are relatively the same stage.

4. Centrifuge at $3,000 \times \mathrm{g}$ for $4 \mathrm{~min}$ at room temperature in order to obtain a loose pellet of adult worms.

5. Using a $10 \mathrm{~mL}$ pipette, carefully remove the supernatant so as to leave the pellet intact. Add $5 \mathrm{~mL}$ of the hypochlorite solution and gently mix.

6. Centrifuge at $3,000 \times g$ at room temperature for 4 minutes.

7. Remove supernatant with a $10 \mathrm{~mL}$ pipette and wash with $7 \mathrm{~mL}$ of $\mathrm{M} 9$ buffer. Repeat this step twice.

\section{Exposing Worms to BPA}

1. After the last wash from step 2.7 above, suspend about $100 \mu \mathrm{L}$ of eggs with M9 buffer. Transfer about $50 \mu \mathrm{L}$ of eggs to $2 \mathrm{~mL}$ microfuge tubes already containing the appropriate concentration BPA diluted in S buffer. Adjust the amount of S-buffer to ensure the correct final concentration of BPA $(0.0 \mu \mathrm{M}, 0.1 \mu \mathrm{M}, 0.5 \mu \mathrm{M}, 1.0 \mu \mathrm{M}, 5.0 \mu \mathrm{M}$ and $10 \mu \mathrm{M})$ per tube. Table 1 illustrates one way to make these dilutions.

\begin{tabular}{|l|l|l|l|}
\hline For Final BPA Concentration & Stock BPA (100 $\mu \mathrm{M})$ & S Buffer & Synchronized Worms \\
\hline $0.1 \mu \mathrm{M}$ & $1 \mu \mathrm{L}$ & $949 \mu \mathrm{L}$ & $50 \mu \mathrm{L}$ \\
\hline $0.5 \mu \mathrm{M}$ & $5 \mu \mathrm{L}$ & $945 \mu \mathrm{L}$ & $50 \mu \mathrm{L}$ \\
\hline $1 \mu \mathrm{M}$ & $10 \mu \mathrm{L}$ & $940 \mu \mathrm{L}$ & $50 \mu \mathrm{L}$ \\
\hline $5 \mu \mathrm{M}$ & $50 \mu \mathrm{L}$ & $900 \mu \mathrm{L}$ & $50 \mu \mathrm{L}$ \\
\hline $10 \mu \mathrm{M}$ & $100 \mu \mathrm{L}$ & $850 \mu \mathrm{L}$ & $50 \mu \mathrm{L}$ \\
\hline
\end{tabular}

Table 1: Stock BPA, S buffer and synchronized worms used to attain the desired concentrations used for our study.

2. Place tubes on a shaker and gently shake for $4 \mathrm{~h}$ at $20^{\circ} \mathrm{C}$, at $25 \mathrm{rpm}$ for a rotary shaker or $25 \mathrm{tilts} / \mathrm{min}$ for a rocking shaker.

3. After $4 \mathrm{~h}$, place tubes in tube holders and allow worms to settle.

4. Discard supernatant and transfer worms to seeded plates (NGM plates with OP50 E. coli). Make the liquid OP50 E. coli culture as stated in 1.5 and seed the plates as stated in 2.1 .

5. Let the worms grow for $60 \mathrm{~h}$ in $20^{\circ} \mathrm{C}$. Examine plates every day for contamination. Contaminated NGM plates are usually discolored and accompanied by individual colonies. Check worms under the microscope for overcrowding. A lack of OP50 E. coli lawn and concentrated worms in small spots signify overcrowding.

\section{Anterior Touch Habituation Assay}

1. Transfer approximately 10 synchronized young adult worms to new unseeded NGM plates using a fire-sterilized $30 \mathrm{G}$ platinum wire pick. Leave worms undisturbed for $5 \mathrm{~min}$ to allow them time to acclimatize to the new plate. 
2. For the habituation response, use an eyebrow hair attached to the end of a wooden skewer or toothpick, and sterilize by dipping in $70 \%$ ethanol. Wipe with a clean lint-free tissue and wait for $1 \mathrm{~min}$ for ethanol to evaporate off. Gently touch the worm on the head (anterior of the pharyngeal bulb) using the eyebrow hair. Repeat the touches, allowing $10 \mathrm{~s}$ in between touches in order to allow the worm to recover.

3. Continue to touch (allowing $10 \mathrm{~s}$ interstimulus intervals) until the worm no longer moves backwards. At this point, the worm has habituated to the stimulus. Record the number of touches required for the worm to habituate.

4. Analyze the difference between the mean habituation rates of the treated and untreated controls using one-way ANOVA followed by Tukey's post hoc test.

\section{Worm Fecundity Assay}

1. Transfer individual L3 worms to a fresh and seeded plate using a platinum pick. Make sure that only one worm is placed per plate. NOTE: After the L3 larval stage, worms develop into L4 larval stage and adulthood. Picking them early is convenient in that they are large enough for the transfer of individual animals while ensuring that they have not already laid any eggs which otherwise would be missed in the count.

2. Count the numbers of eggs laid every $12-24 \mathrm{~h}$. After counting, transfer the parent worm to a new plate. Repeat until the worm stops laying eggs, typically after 5 days when incubated at $20^{\circ} \mathrm{C}$

NOTE: On average, a wild type worm can lay up to 300 eggs; therefore, transferring the parent to a new plate daily prevents overcrowding.

3. Analyze the difference between the average number of eggs laid by the treated and untreated controls using one-way ANOVA followed by Tukey's post hoc test.

\section{Representative Results}

Earlier studies with $C$. elegans have reported that continual exposure to high BPA concentrations ( $\geq 1 \mathrm{mM})$ throughout embryo development and adulthood decreases fecundity ${ }^{14}$. Subsequent studies reported from our lab have shown that embryos that have been exposed to BPA for a limited period of $4 \mathrm{~h}$ in the beginning stages of their development showed a decrease in the number of viable eggs laid as adults ${ }^{15}$ (Figure 2). Two key features of the methodology were that (i) the BPA concentrations used were significantly lower $(0.1 \mu \mathrm{M}$ to $10 \mu \mathrm{M}$ range) and (ii) the exposure was limited to the early embryonic period. Besides decreased fecundity even at lower doses, our habituation assays revealed that worms exposed to BPA as embryos required more stimuli to become habituated when compared to worms exposed to vehicle alone ${ }^{15}$ (Figure 3). These effects are noteworthy in that they are based on low-dose BPA exposure, following the rationale that subtler effects on neuronal function that may not be morphologically apparent are likely to be discernible through behavioral changes. In short, the representative results presented here underscore the fact that the detrimental effects of BPA exposure to an embryo impacts its development, including neuronal function that can be assessed through adult behavioral assays in $C$. elegans. Protocols provided here can be used for testing low-dose as well as long-term effects of other potential toxicants including endocrine disruptive compounds.

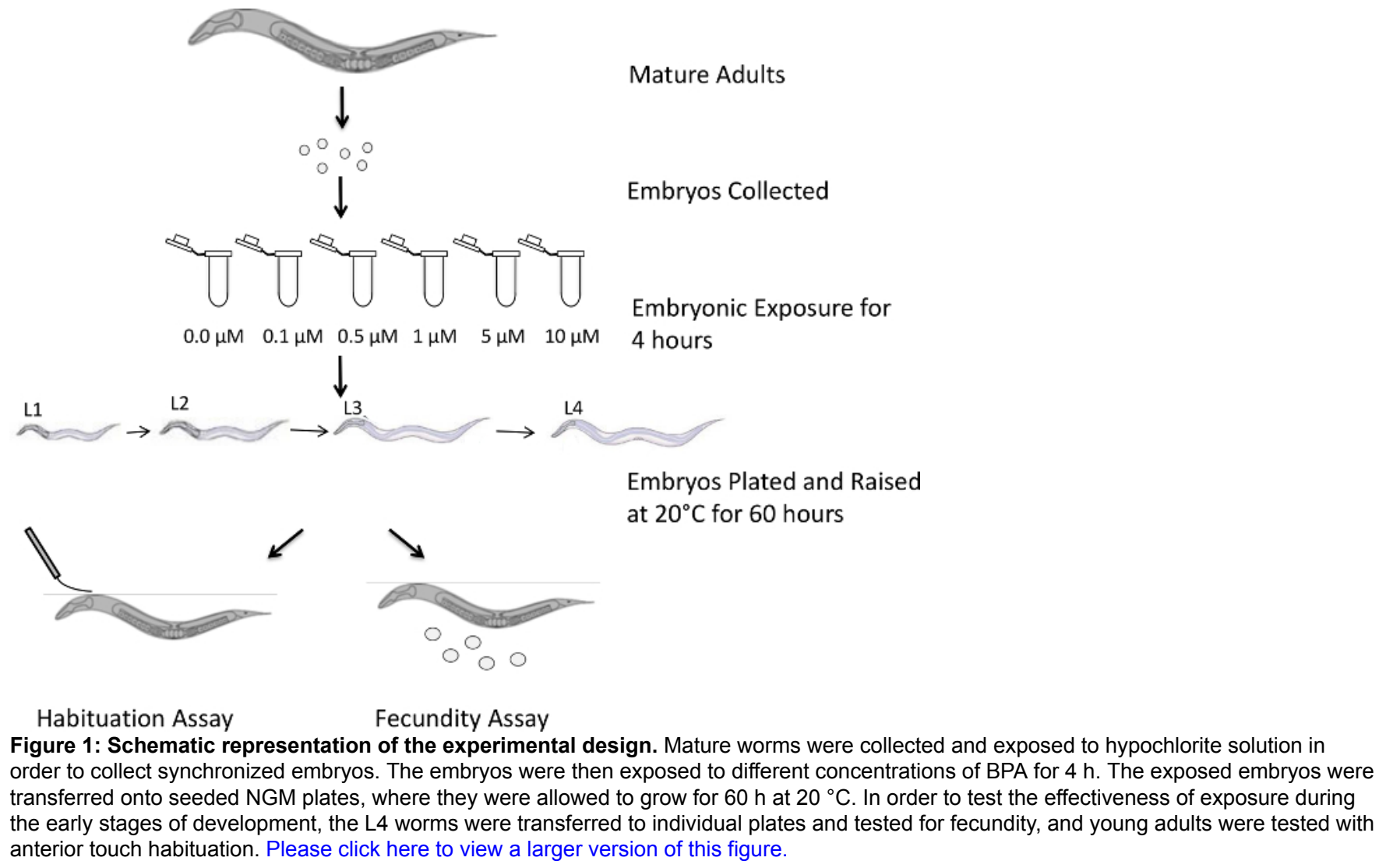




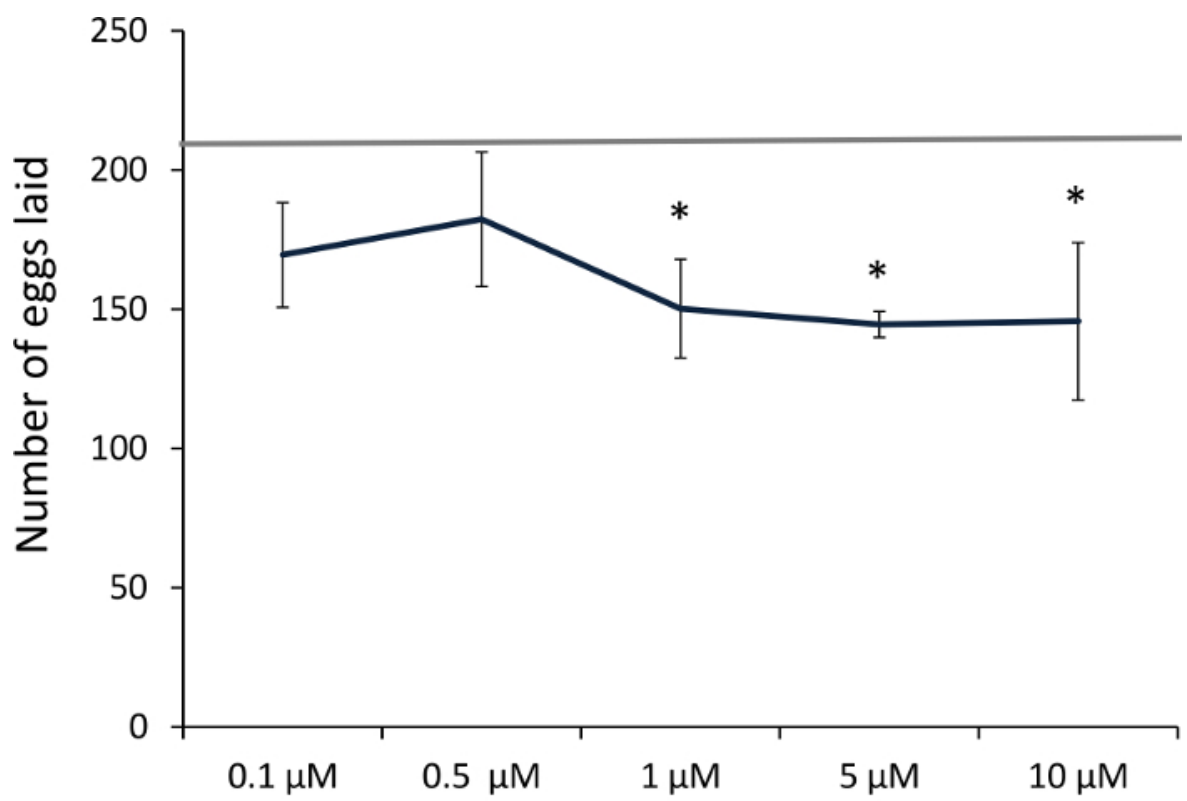

\section{BPA Concentration}

Figure 2: Fecundity is lowered by BPA. Exposure to $1 \mu \mathrm{M}$ and higher concentrations of BPA significantly decreased the number of eggs laid compared to the control (represented by the horizontal line; $n=10,{ }^{*} p<0.05$ ). Error bars denote SEM. Analysis done using ANOVA, Tukey's post hoc test. This figure has been modified from Mersha et al., $2015^{15}$. Please click here to view a larger version of this figure.

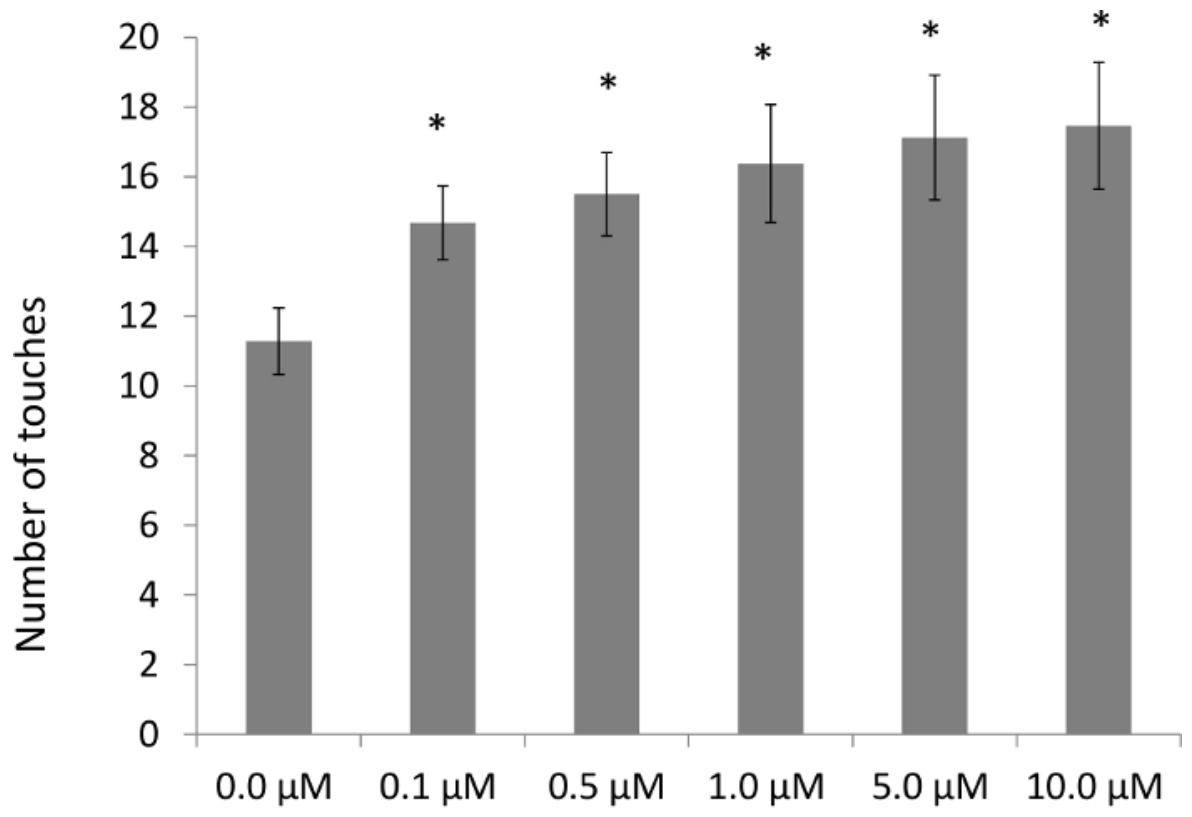

BPA Concentration

Figure 3: Long-term effects of embryonic BPA exposure are reflected in adult behavior. Embryos exposed to BPA concentrations as low as $0.1 \mu \mathrm{M}$ affects non-associative anterior touch habituation in adults $\left(n=60,{ }^{*} p<0.05\right)$. Error bars denote SEM; ANOVA, Tukey's post hoc test. This figure has been modified from Mersha et al., $2015^{15}$. Please click here to view a larger version of this figure.

\section{Discussion}

Phases of rapid growth and development such as embryogenesis are particularly vulnerable to the detrimental effects of various endocrinedisrupting compounds, including BPA. We provide detailed protocols for studying the effects of exposure to BPA or other toxicants in the $C$. elegans invertebrate model, which allows convenient titration of a range of concentrations to assess its effect on embryo viability (Figure 2). Follow up behavioral experiments on surviving adults that develop from embryos that were exposed to low doses of BPA allow assaying longterm effects on neuronal function (Figure 3). The central idea of this paper is to provide detailed protocols for exposing embryos to various toxicants during the early embryogenesis period in the C. elegans model; the first $4 \mathrm{~h}$ window of its 11.5 hour embryonic development period 
(at $20^{\circ} \mathrm{C}$ ) corresponds to the proliferation period, which is followed by organogenesis. Towards this end, we have provided validation of the end points through fecundity and non-associative learning assays. While the mechanistic basis of the action of BPA and other bisphenols is the holy grail towards which research in this field is aimed, we have not attempt to offer a mechanistic explanation for the action of BPA in this methodology focused article. However, we would like to point out that the $C$. elegans model offers an ideal system to further dissect the mechanism of action. For example, in future work on deciphering mechanisms of BPA action, $C$. elegans mutants can be generated and screened for a resistance to the effects of BPA and thus pave the way for deciphering the pathway. Additionally, our companion paper deals with the effect of BPA at the neural network synchrony level in vertebrates, providing another avenue to understand the basis for investigating potential mechanisms for toxicant effects ${ }^{19}$.

In the methodology detailed in this paper, some of the critical steps that should be executed extra carefully include the following: Choosing a plate with well-fed $C$. elegans adults with mature eggs visible under the dissection microscope is crucial, and failing to follow this step will result in an insufficient sample size of embryos for the EDC exposure experiment. It is also important to make sure that the hypochlorite solution is made fresh to avoid obtaining an unsynchronized population that will interfere with the ability to perform BPA exposure during early embryogenesis. Washing pellets with M9 (after hypochlorite exposure) is also a very important step. If not done properly, the high concentration of bleach might result in dead embryos. Additionally, embryos should be transferred to seeded NGM plates after $4 \mathrm{~h}$. If left in the BPA solution for an extended period of time, the embryos are likely to develop into the long-lived dauer larvae. This will significantly interfere with both fecundity assay and behavioral assays. If any of these steps is missed, it is advisable to start the procedure from the very beginning. If for any reason, the NGM plate with worms is contaminated or does not have enough food, it will add stress on the worms thereby skewing the data collected. Such plates should be discarded and not be used in the experiment.

Previously, a remarkable study successfully used the $C$. elegans model to demonstrate that chromosome abnormalities caused by BPA result due to the breakdown of DNA repair machinery in the germline ${ }^{14}$. However, it is noteworthy that the above study design had used continuous BPA exposure of $\mathrm{C}$. elegans to high doses of BPA throughout its lifespan. Our methodology was designed to study low doses of BPA exposure during early embryogenesis, so as to assay its effects on embryonic viability and subtler long-term effects on the survivors. To our knowledge, no methodology has been developed to expose early stage embryos to very low doses of BPA limited to a specified period of time; thus, making the method presented here novel.

In addition, protocols provided here can be easily adapted to study the effects of other EDCs during the proliferation phase of embryogenesis However, in order to study late embryonic stages, the protocol will require key modifications to the critical time window of development under focus. Furthermore, our protocol will not be suitable for experiments requiring longer times of toxicant exposure, as it opens up the possibility of the alternate development pathway resulting in the long-lived dauer arrest at the second molting cycle, which is resistant to harsh conditions ${ }^{20}$. Further modifications and refinements will be needed for longer exposure times through supplement with a food source that does not have potential to metabolize the toxicant or EDC e.g. autoclaved E. coli. In conclusion, our methodology can be utilized to assess the effects of various endocrine disrupting chemicals on the fecundity and on neuronal function in the invertebrate model system $C$. elegans.

\section{Disclosures}

The authors declare that they have nothing to disclose.

\section{Acknowledgements}

Support by NSF (EPSCoR EPS-0814251) and NIH (1P20GM103653-01A1) to MKT and HSD, Delaware State University's institutional student support to MDM (Title III HBGI) and KRS (NIH-INBRE) is gratefully acknowledged. Thanks to the C. elegans Genetic Center (supported by NIH Office of Research Infrastructure Programs P40 OD010110) for C. elegans wild-type N2 strain.

\section{References}

1. Gore, A. C. et al. Executive Summary to EDC-2: The Endocrine Society's Second Scientific Statement on Endocrine-Disrupting Chemicals. Endocr. Rev. 36, 593-602 (2015).

2. Braun, J. M. Early-life exposure to EDCs: role in childhood obesity and neurodevelopment. Nat. Rev. Endocrinol. 13, 161-173 (2017).

3. Melnik, R. et al. Summary of the National Toxicology Program's Report of the Endocrine Disruptors Low-Dose Peer Review. Environ. Health Perspect. 110, 427-431 (2002).

4. Kortenkamp, A. Ten years of mixing cocktails: a review of combination effects of endocrine-disrupting chemicals. Environ. Health Perspect. 115 Suppl 1, 98-105 (2007).

5. Vandenberg, L. N. et al. Hormones and endocrine-disrupting chemicals: low-dose effects and nonmonotonic dose responses. Endocr. Rev. 33, 378-455 (2012).

6. Eramo, S., Urbani, G., Sfasciotti, G. L., Brugnoletti, O., Bossu, M., \& Polimeni, A. Estrogenicity of bisphenol A released from sealants and composites: a review of the literature. Ann Stomatol. (Roma). 1, 14-21 (2010).

7. Cantonwine, D. E., Ferguson, K. K., Mukherjee, B., McElrath, T. F., \& Meeker, J. D. Urinary Bisphenol A Levels during Pregnancy and Risk of Preterm Birth. Environ. Health Perspect. 123, 895-901 (2015).

8. Vandenberg, L. N., Maffini, M. V., Sonnenschein, C., Rubin, B. S., \& Soto, A. M. Bisphenol-A and the great divide: a review of controversies in the field of endocrine disruption. Endocr. Rev. 30, 75-95 (2009).

9. Suzuki, T. et al. Prenatal and neonatal exposure to bisphenol-a enhances the central dopamine d1 receptor-mediated action in mice: enhancement of the methamphetamine-induced abuse state. Neuroscience. 117, 639-644 (2003).

10. Rosenfeld, C. S. Bisphenol A and phthalate endocrine disruption of parental and social behaviors. Front. Neurosci. 9, 1-15 (2015).

11. Patisaul, H. B., \& Adewale, H. B. Long-term effects of environmental endocrine disruptors on reproductive physiology and behavior. Front. Behav. Neurosci. 3, 10 (2009). 
12. Lee, D., \& Jacobs, D. R., Jr. Methodological issues in human studies of endocrine disrupting chemicals. Rev. Endocr. Metab. Disord. 16, 289-297 (2015).

13. Mimoto, A. et al. Identification of an estrogenic hormone receptor in Caenorhabditis elegans. Biochem. Biophys. Res. Commun. 364, 883-888 (2007).

14. Allard, P., \& Colaiacovo, M. Bisphenol A impairs the double-strand break repair machinery in the germline and causes chromosome abnormalities. Proc. Natl. Acad. Sci. U.S.A. 107, 20405-20410 (2010).

15. Mersha, M. D., Patel, B. M., Patel, D., Richardson, B. N., \& Dhillon, H. S. Effects of BPA and BPS exposure limited to early embryogenesis persist to impair non-associative learning in adults. Behav. Brain. Funct. 11, 27 (2015).

16. Chen, Y. et al. Exposure to the BPA-Substitute Bisphenol S Causes Unique Alterations of Germline Function. PLoS Genet. 12, e1006223 (2016).

17. Rankin, C. H. et al. Habituation revisited: an updated and revised description of the behavioral characteristics of habituation. Neurobiol. Learn. Mem. 92, 135-138 (2009).

18. Hope, I. C. elegans: A Practical Approach. Oxford University Press (1999).

19. Sanchez, K., Mersha, M., Dhillon, H. S., \& Temburni, M. Assessment of the Effects of Endocrine Disrupting Compounds on the Development of Vertebrate Neural Network Function Using Multi-electrode Arrays. J. Vis. Exp. In Press (2017).

20. Hu, P. J. in WormBook The C. elegans Research Community. wormbook, (2007). 